\title{
First i-TED demonstrator: a Compton imager with Dynamic Electronic Collimation
}

\author{
V. Babiano, J. Balibrea, L. Caballero, D. Calvo, I. Ladarescu, J. Lerendegui, S. Mira Prats, C. Domingo-Pardo ${ }^{1}$ \\ Instituto de Física Corpuscular, CSIC-University of Valencia, Spain
}

\begin{abstract}
i-TED consists of both a total energy detector and a Compton camera primarily intended for the measurement of neutron capture cross sections by means of the simultaneous combination of neutron time-of-flight (TOF) and $\gamma$ ray imaging techniques. TOF allows one to obtain a neutron-energy differential capture yield, whereas the imaging capability is intended for the discrimination of radiative background sources, that have a spatial origin different from that of the capture sample under investigation. A distinctive feature of i-TED is the embedded Dynamic Electronic Collimation (DEC) concept, which allows for a trade-off between efficiency and image resolution. Here we report on some general design considerations and first performance characterization measurements made with an i-TED demonstrator in order to explore its $\gamma$-ray detection and imaging capabilities.
\end{abstract}

Keywords: Compton imaging; position-sensitive detectors; monolithic crystals; Silicon photomultiplier;

\section{Introduction}

Neutron capture cross sections are a key nuclearphysics ingredient for the study of the formation of elements heavier than iron in the stars [1, 2, 3]. Direct $(n, \gamma)$ measurements are particularly relevant for the so-called slow nucleosynthesis $(s-)$ process, where most of the involved nuclei can be accessed in laboratory experiments. Presently, the main fronts of experimental research in this field focus, on one hand, on new measurements targeting accuracies of $\lesssim 5 \%$ required by the models [4, 5] and, on the other hand, on the first measurement of radioactive $s$ process branching nuclei, which are particularly relevant for benchmarking the physical conditions inside stars [3].

Neutron activation and time-of-flight (TOF) are the two main methodologies used to determine neutron capture cross sections in the relevant stellar energy range (see e.g. [3]). The TOF approach offers the advantage of being a general technique applicable to any isotope, provided that sufficient sample mass becomes available. It also allows one to cover the full stellar energy regime in one single measurement. For TOF experiments essentially two different $\gamma$-ray detection apparatus are utilized: Total Absorption Calorimeters (TACs) [6, 7, 8] and TotalEnergy Detectors (TEDs) [9, 10, 11]. TEDs have been used since many years for neutron capture cross section measurements of astrophysical interest, including experiments with radioactive samples of $s$-process branching nuclei [12, 13, 14]. However, one limitation of state-ofthe-art TEDs [15] arises from neutrons scattered in the capture-sample, and subsequently thermalized and captured in the surrounding walls and structural materials in the measuring station. This effect has been investigated in detail in Ref. [16] and, in some important cases (see e.g. [17, 18]), it fully dominates the background level already beyond a few $\mathrm{keV}$ of neutron energy. This is precisely the energy range of interest for nucleosynthesis studies in low-mass AGB stars during H-burning $(8 \mathrm{keV})$ and He-burning $(23 \mathrm{keV})$, and also during core He-burning $(26 \mathrm{keV})$ and shell C-burning $(91 \mathrm{keV})$ in massive stars [3]. Heavy lead shieldings (see e.g. Fig. 3 in Ref. [19]) or mechanical collimators [20] become pro- 
hibitive owing to the increased neutron scattering and capture in the shielding itself, which rather amplifies the problem of the neutron-induced $\gamma$-ray background.

One alternative to this problem is to use electronic collimation [21, 22], which is based on the Compton scattering law. In the framework of the HYMNS project [23] we are developing an electronically collimated TED to be applied for neutron capture TOF measurements. As described in Ref. [24] this approach would allow one, on an event-by-event basis, to infer information on the incoming radiation direction, thereby enabling the possibility to distinguish between true capture $\gamma$-rays coming from the sample and contaminant $\gamma$-rays induced by neutroncapture in the surroundings of the detection set-up. Similar concepts have been exploited in other fields, such as $\gamma$-ray astronomy [25, 26, 27], medicine [28] or nuclear structure [29] but thus far, it has never been attempted in the field of TOF neutron-capture measurements. In this work we present a detection system, which represents a first demonstrator of the so-called Total-Energy Detector with $\gamma$-ray imaging capability (i-TED) [24]. The suitability of i-TED as a total-energy detector has been discussed on the basis of MC simulations in Ref. [24] and an experimental study using a neutron beam will be reported in a separate article. In this work we focus on the suitability of i-TED as a $\gamma$-ray imager. General considerations related to the Compton camera design are discussed in Sec. 2. Sec. 3 describes the technical aspects of the first assembled i-TED demonstrator. Measurements made in the laboratory to characterize the efficiency and resolution are discussed in Sec. 4. Finally, Sec. 5 summarizes the main conclusions and next steps.

\section{Dynamic Electronic Collimation}

Detection efficiency and image resolution are two of the most relevant performance aspects of any Compton imager. These two elements are discussed in the following, with the aim of introducing the concept of Dynamic Electronic Collimation (DEC).

In Compton cameras based on two detection planes the intrinsic $\gamma$-ray detection efficiency is determined by the efficiency of its scatter $(S)$ and absorber $(A)$ detectors and by the distance between them, hereafter indicated by $d_{f}$. The subindex $f$ is used here to reflect that the $S-A$ separation length, $d_{f}$, may be used to "focus" or tune the sys- tem, and thus adjust the interplay between image resolution and efficiency, as it is discussed below.

Assuming that the energy $E$ of the incident $\gamma$-ray is known, the uncertainty on the Compton angle $\delta \theta$ can be approximated by the following analytic expression (see Ref. [30]),

$$
\delta \theta=\frac{1}{\sin \theta}\left(\left(\frac{m_{e} c^{2}}{E^{\prime 2}}\right)^{2}\left(\frac{\delta E^{\prime}}{E^{\prime}}\right)^{2}+2 \sin ^{2} \theta\left(\frac{\delta r}{r}\right)^{2}\right)^{1 / 2}
$$

where $\theta$ is the Compton angle, $m_{e}$ is the rest mass of the electron, $c$ is the speed of light, $r$ is the distance between the first and the second interactions, and $E^{\prime}$ is the energy of the scattered $\gamma$-ray. Uncertainties on these quantities are indicated by the prefix $\delta$. Obviously, the first parameters to optimize in a Compton imager are the energyresolution $\delta E$ and the spatial-resolution $\delta r$, because low uncertainties on these magnitudes lead to high angular resolution and imaging capabilities. In order to gain insight into this aspect, let us assume that the $\gamma$-ray source is sufficiently far from the detector, so that the distance between the first and the second interactions can be approximated by $r \simeq d_{f} / \cos (\theta)$. With such approximation one can illustrate in a simple manner the overall trend of the Compton angle uncertainty, or at least an upper limit for it. Thus, the uncertainty on the Compton angle $\delta \theta$ can be graphically displayed as a function of the $S-A$ separation $d_{f}$, as demonstrated in Fig. 1 for a $\gamma$-ray source of $662 \mathrm{keV}$. The uncertainty $\delta \theta$ is shown in color scale for a series of spatial resolutions $\delta r=$ $1 \mathrm{~mm}$ [31, 32], $3 \mathrm{~mm}$ [33, 34, 35, 32] FWHM and energyresolutions $\delta E^{\prime} / E^{\prime}=3.5 \%, 6.5 \%$ FWHM at $662 \mathrm{keV}$, representative of high-resolution inorganic scintillation crystals (see Ref. [36] and references therein). For the graphical representation the value of $E^{\prime}$ in eq. (1) was calculated using the Compton law for each scattering angle $\theta$. For the relative energy resolution $\delta E^{\prime} / E^{\prime}$ a $1 / \sqrt{E}$ dependence was assumed with respect to the values quoted at $662 \mathrm{keV}$. The Doppler contribution to $\delta E^{\prime}$ due to the momentum of the target electron [37] can be fully neglected because it is much smaller than the intrinsic energy resolution of the inorganic crystals used in this work.

Regarding the quoted resolutions on $\delta E$ and $\delta r$, clearly, a good energy resolution (3.5\% instead of $6.5 \%$ ) has a large impact on the attainable angular (image) resolution, 

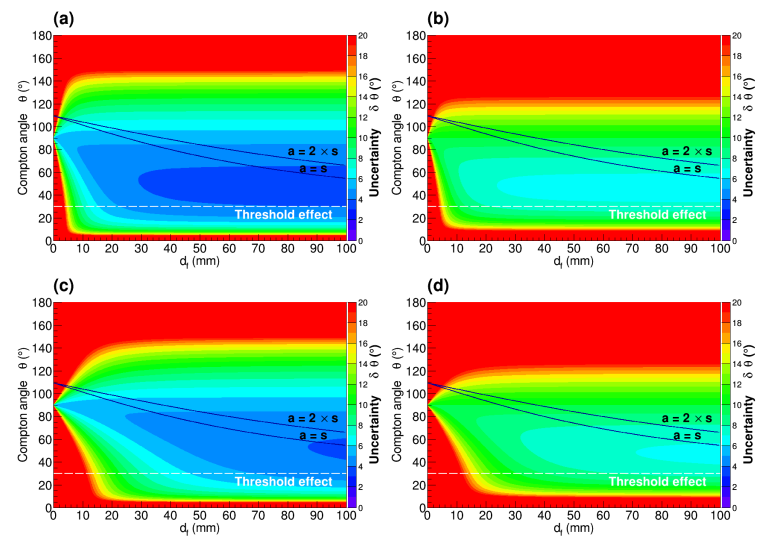

Figure 1: Angular resolution calculated for a Compton camera as a function of the distance $d_{f}$ between scatter and absorber detectors. From top to bottom the spatial and energy resolutions (FWHM) are of (a) $1 \mathrm{~mm}$, $3.5 \%$, (b) $1 \mathrm{~mm}, 6.5 \%$, (c) $3 \mathrm{~mm}, 3.5 \%$ and (d) $3 \mathrm{~mm}, 6.5 \%$. See text for details.

as it can be appreciated comparing Fig 1 (a) and (c) with Fig 1 (b) and (d). On the other hand, the intrinsic spatial resolution of the radiation detectors has a lesser impact on the attainable angular resolution $\delta \theta$. This effect can be observed by the small changes in $\delta \theta$ when the spatial resolution varies from $1 \mathrm{~mm} \mathrm{Fig} 1$ (a) and (b) to $3 \mathrm{~mm}$ Fig 1 (c) and (d). The latter statement is particularly clear for sufficiently large $S$-A separation lengths of $d_{f} \gtrsim 30 \mathrm{~mm}$.

For $\gamma$-ray energies different from $662 \mathrm{keV}$ the general pattern of Fig. 11 remains rather similar, with the best (minimum) value of $\delta \theta$ changing from Compton angles of $\theta \sim 45^{\circ}$ at $662 \mathrm{keV}$ (Fig. (1) down to $\theta \sim 40^{\circ}\left(20^{\circ}\right.$ ) at $1 \mathrm{MeV}(5 \mathrm{MeV})$, and to $\theta \sim 70^{\circ}$ at $150 \mathrm{keV}$. Thus, for each incident $\gamma$-ray energy there is a small shift between the Compton angle for the best angular resolution and the Compton angle corresponding to the maximum probability of the Klein-Nishina distribution [38] (see Fig. 22. Nevertheless, both follow the same trend with the $\gamma$-ray energy, which is a positive feature in terms of image reconstruction.

In general, Compton angles around $0^{\circ}$ and $180^{\circ}$ lend to poor angular resolutions owing to the $1 / \sin (\theta)$ factor in eq. (1). The contribution of the first term in eq. (1) increases with the inverse value of the scattered $\gamma$-ray energy $E^{\prime}$ to the fourth power. Hence, due to the Compton scattering law and the energy dependency of the resolu-

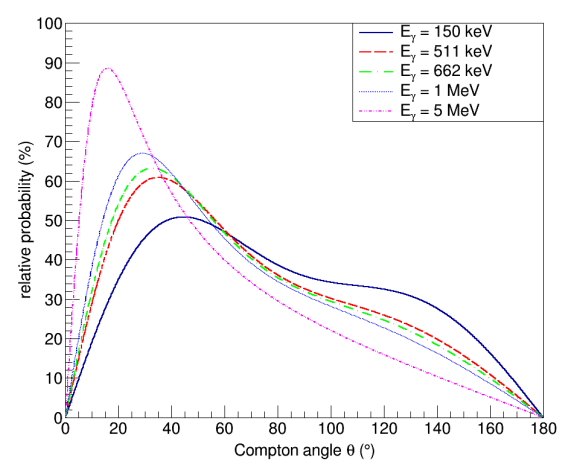

Figure 2: Relative scattering probability as a function of the Compton angle for a series of $\gamma$-ray energies between $150 \mathrm{keV}$ and $5 \mathrm{MeV}$.

tion, the angular resolution worsens significantly above $\theta \sim 140^{\circ}$, or even $\sim 120^{\circ}$, depending on the intrinsic energy resolution of the detector (see Fig. 11).

On the other hand, the low range of scattering angles $10^{\circ} \lesssim \theta \lesssim 120^{\circ}\left(140^{\circ}\right)$ represents the most valuable contribution in terms of image reconstruction (see Fig. 11). In this high-resolution angular range $\left(10^{\circ} \lesssim \theta \lesssim 120^{\circ}\right)$ the second term in eq. (1) contributes most, thus allowing one to enhance the angular resolution by means of increasing the $S$-A separation or $d_{f}$ length. Because there is also a strong dependency between $\gamma$-ray efficiency and $d_{f}$ distance, the latter can be adjusted for a trade-off between detection efficiency and image resolution, or for maximizing either of them. It is also worth noting that, for the quoted intrinsic position- and energy-resolutions, the maximal useful $S-A$ separation $d_{f}$ is of about $40-60 \mathrm{~mm}$ in all cases. Indeed, beyond that distance there is essentially no gain in resolution and only a strong decrease in efficiency would be obtained.

The concept of Dynamic Electronic Collimation (DEC) [39] enhances the fieldability and applicability scope of the Compton camera, when compared to a system where both $S$ - and $A$-detection planes are placed at a fixed distance. Several previous studies have reported on the impact that the $S-A$ distance has on angular resolution [40, 41] and on both angular resolution and efficiency [42, 43, 44, 45], thereby focusing mainly on optimizing the detector geometry. More recently, the suitability of a Compton system with manually 
adjustable $S$ - $A$ separation has been demonstrated in the field of 3D molecular Compton imaging by the group of Tokyo [44, 46]. With the aim of taking full advantage of the DEC concept, the i-TED Compton imager presented in this work incorporates an embedded micro-positioning drive with a large range of $50 \mathrm{~mm}$, which enables for a variable and accurate separation of the $S-A$ detectors distance $d_{f}$. More technical details are given below in Sec. 3

The main advantage of DEC for the measurement of neutron capture cross sections is envisaged for the reduction of neutron-induced background radiation. Indeed, in neutron TOF measurements the signal-to-background ratio typically decreases with increasing neutron energy due to the $1 / v$ dependency of the cross section and the relatively flat background rate (see for example Fig. 6 in Ref. [16]). Thus, it may become possible to sample a broad neutron energy range using different values of $d_{f}$, which are adjusted to each specific neutron-energy decade or energy interval for an optimal background discrimination and sufficient counting statistics.

The DEC-technique may be also of interest in other fields such as homeland security scenarios, decommissioning and nuclear waste monitoring. Given the variety of $\gamma$-ray emitters, activities and spatial distributions, a first inspection using a short $d_{f}$ may be useful for a rapid survey to roughly identifiy the main radionuclides and their coarse spatial origin. Once this information has been processed, if needed, a more detailed image can be obtained aftewards using a larger $d_{f}$ value. DEC will be further discussed in Sec. 4 on the basis of dedicated laboratory measurements.

Finally, there are two experimental effects which constrain remarkably the angular resolution displayed in Fig. 1. They are discussed in the following two sections.

\section{Geometry constraints}

The geometry of the detection setup determines to a large extent the total detection efficiency, but it also constrains the maximum Compton angle that can be measured. This is schematically illustrated in Fig. 3 for a simplified model based on two parallel aligned squareshaped detectors. Assuming $S$ - and $A$-detectors of sizes $s$ and $a$, respectively, the maximum measurable Compton

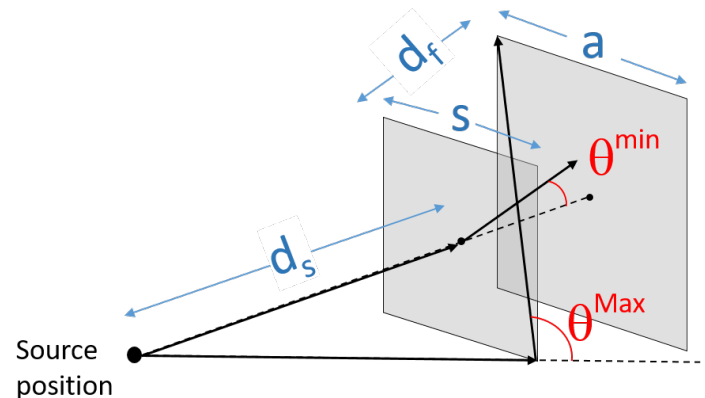

Figure 3: Schematic representation of a generic Compton camera. The maximum and minimum Compton angles are represented for a $\gamma$-ray source centered at a distance $d_{s}$ from the Camera. See text for details.

angle for a centered source at a distance $d_{s}$ is given by,

$$
\theta^{\text {Max }}=\arctan \left(\frac{s}{\sqrt{2} \cdot d_{s}}\right)+\arctan \left(\frac{a+s}{\sqrt{2} \cdot d_{f}}\right) .
$$

Note that in the latter equation, both terms cannot be grouped in one single term using the arctangent addition formula $\arctan (u) \pm \arctan (v)=\arctan ((u \pm v) /(1 \mp u \cdot v))$ because the latter holds only for $u \cdot v<1$. The value of $\theta^{M a x}$ is displayed in Fig. 4 in color scale as a function of $d_{f}$ and the distance to a centered point-like source $d_{s}$. Two different cases are considered here. Fig. 4.(a) shows the expected behavior for a Compton camera where both S- and A-detectors are squared and have the same size $a=s=50 \mathrm{~mm}$. Fig. 4.(b) shows the maximum measurable Compton angle when the size of the A-detector is two times the size of the $S$-detector $(a=2 \times s=100 \mathrm{~mm})$.

For a $\gamma$-ray source located at a short distance $\left(d_{s} \sim\right.$ $\mathrm{cm}$ ) practically all relevant (high resolution) Compton angles are available over the main range of $S-A$ separation lengths $d_{f}=10-60 \mathrm{~mm}$. For sources at distances of $d_{s} \sim 10 \mathrm{~cm}$, the maximum measurable Compton angle is constrained to $\theta^{\operatorname{Max}} \lesssim 80^{\circ}$. This constraint represents already a significant loss in terms of usable Compton angles. Such $\theta^{\text {Max }}$ limit for a source at $d_{s}=10 \mathrm{~cm}$ is shown in Fig. 1 for the $s=a$ and $a=2 \times s$ configurations as a function of $d_{f}$. It is worth noting that this is a purely geometrical constraint, which does not depend on the $\gamma$ ray energy. For the location of distant sources, $d_{s} \gtrsim 1 \mathrm{~m}$, using a $d_{f}=50 \mathrm{~mm}$ the maximum detectable Compton 

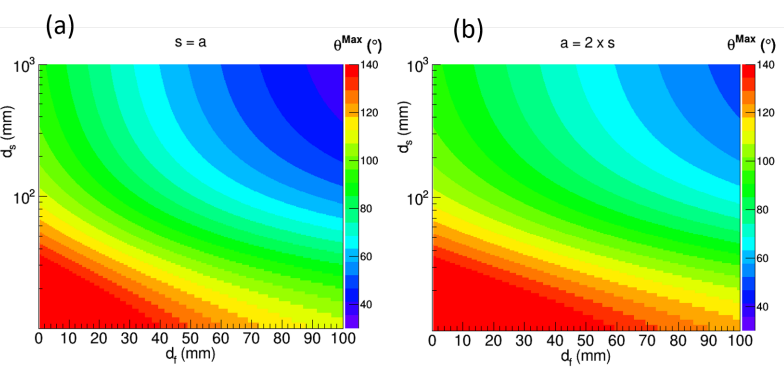

Figure 4: Representation of the maximum measurable Compton angle $\theta^{M a x}$ with a color scale (in degrees) for a generic Compton camera based on squared detectors arranged in parallel configuration. Figure (a) shows the behavior for a camera based on $\mathrm{S}$ - and A-detectors of equal size ( $s=a=50 \mathrm{~mm}$ ). Figure (b) corresponds to a configuration where the absorber detector has two times the size of the scatter detector ( $a=$ $2 \times s=100 \mathrm{~mm}$ ). See text for details.

angle $\theta^{\text {Max }}$ becomes $\sim 60^{\circ}$ and $\sim 80^{\circ}$ for the $s=a$ and $a=2 \times s$ configurations, respectively. Thus, a large absorber plane seems particularly interesting for the measurement of low energy distant sources (see also Fig. 2). A significant portion of usable Compton angles are available yet below $\theta^{\text {Max }}=60^{\circ}-80^{\circ}$ over a broad range of energies (see Fig. 2). However, the low angular range is constrained by another experimental effect, which is described below.

\section{Threshold effect}

A further ineluctable experimental effect, which impacts the performance of the Compton camera is the (noise-rejecting) threshold in the Scatter detector. Due to the Compton scattering law, a cut in deposited energy is directly translated into a threshold for the lowest measurable Compton angle. For $\gamma$-quanta of $662 \mathrm{keV}$ this relationship is illustrated in Fig. 5. A threshold of $100 \mathrm{keV}$ in the $S$-detector therefore leads to a bottom limit on the detectable angle of $\theta_{\min } \sim 30^{\circ}$. Such threshold constraint is also indicated by dashed lines in Fig. 1

In summary, due to the aforementioned geometry- and threshold effects, only the central region of Compton angles becomes experimentally accessible (see Fig. 1). Although this statement strictly holds only for a centered $\gamma$-ray source and the simple geometric model used, it serves to provide a realistic idea about some of the main constraints in the design of a Compton camera. For a

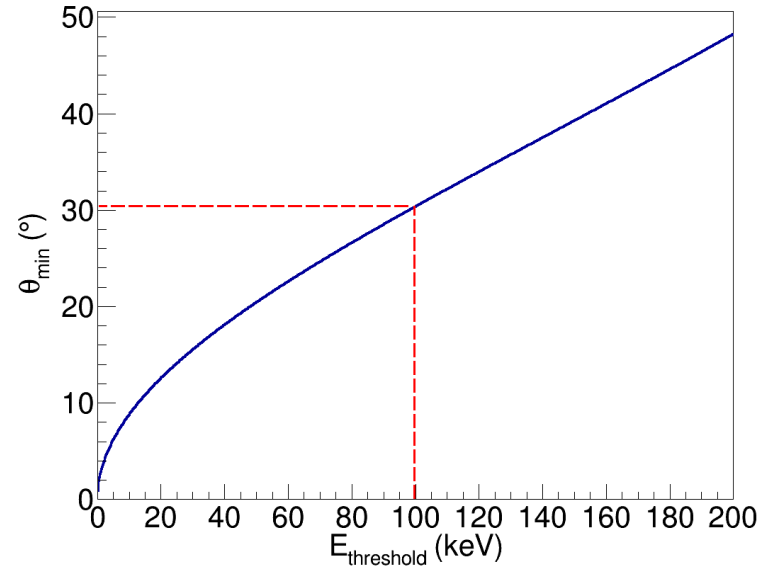

Figure 5: Relationship between the energy threshold in the scatter detector and the minimum measurable Compton angle.

$100 \mathrm{keV}$ noise-rejecting threshold in the S-detector and the $a=2 \times s$ configuration, the range of measurable angles is constrained to $30^{\circ} \lesssim \theta \lesssim 80^{\circ}-100^{\circ}$ for $662 \mathrm{keV}$ $\gamma$-rays depending on $d_{f}$. The next sections present the assembly and realization of a Compton imager built according to the concepts discussed above.

\section{Materials}

i-TED is based on the Compton camera design with the aforementioned $a=2 \times s$ configuration. The positionsensitive scatter detector $(\mathrm{S})$ consists of a $50 \times 50 \times 10 \mathrm{~mm}^{3}$ $\mathrm{LaCl}_{3}(\mathrm{Ce})$ monolithic crystal optically coupled with silicon grease (Saint Gobain BC-630) to a silicon photomultiplier SiPM (SensL ArrayJ-60035-64P-PCB), which features a segmentation of $8 \times 8$ pixels. The absorber detector is based on an array made from four co-planar $\mathrm{LaCl}_{3}(\mathrm{Ce})$ blocks, each of them with a size of $50 \times 50 \times 25 \mathrm{~mm}^{3}$ $\mathrm{LaCl}_{3}(\mathrm{Ce})$ and readout by the same type of SiPM sensors. Thus, the full detection system comprises 320 channels, which are readout by means of front-end and processing PETsys FEB/D-v2 electronics [47]. Thermal stabilization of the ASICs is accomplished by means of $20 \times 20 \mathrm{~mm}^{2}$ Peltier cells (FPH1-7106NC) thermally coupled to the external chip surface by means of non-silicone heat transfer compound (HTCP20S from Electrolube). In order to op- 
timize cooling performance, the hot side of the Peltier cell is also thermally coupled to a small metallic heatsink assembled to a mini DC-axial fan (MC36358 from Multicomp), which helps to dissipate heat efficiently. Further details about the instrumentation related to the position sensitive detectors can be found in Ref. [32]. A picture of the whole assembled detection system is shown in Fig. 6

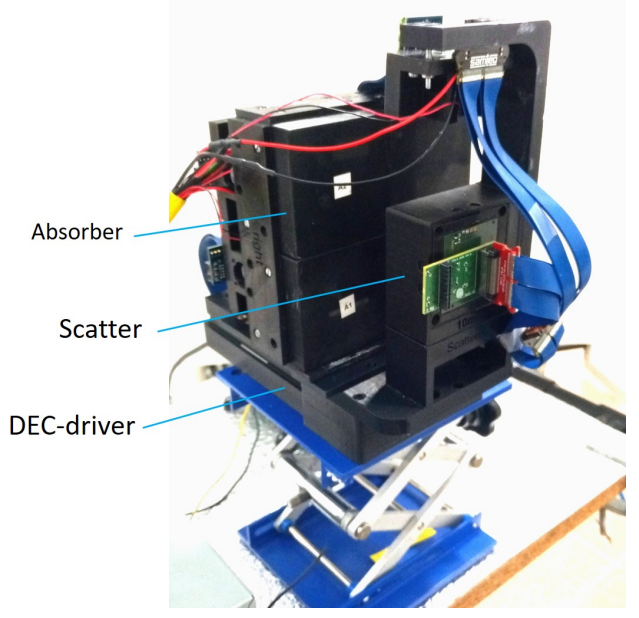

Figure 6: The i-TED Compton camera equipped with one scatter and four absorber detectors mounted on a positioning drive for the implementation of the DEC-technique. See also Fig.7

Dynamic Electronic Collimation (see Sec. 2) is accomplished in i-TED by means of a micropositioning stage (M-683 from PI-miCos) mounted underneath the array of A-detectors. This stage has a load capacity of $50 \mathrm{~N}$ and includes an integrated linear encoder with a resolution of $0.1 \mu \mathrm{m}$. The embedded piezoceramic linear motor (PILine U-164) allows one to remotely control the position of the absorption layer with respect to the scattering plane with a sub-micrometric precision over a range of $50 \mathrm{~mm}$. Communication with the external computer is made by means of a controller (C-867 from PI-miCos) via USB connection. Fig. 7] shows a picture of the full set-up with the $A$-detection layer taken appart to illustrate better the different components. The information about the distance $d_{f}$ between the $S$ - and $A$-layers is directly fed into our data-stream and afterwards used for the image reconstruction (see Sec. 4).

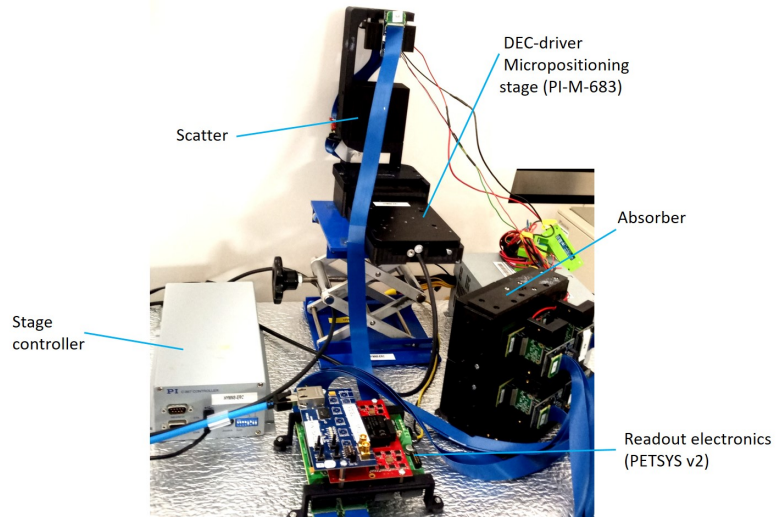

Figure 7: i-TED with A-layer taken off to show the positioning drive and other components.

\section{Measurements and results}

An energy calibration of each detector is performed in the full energy range from $122 \mathrm{keV}$ up to $1410 \mathrm{keV}$ using a radioactive source of ${ }^{152} \mathrm{Eu}$. The energy resolution at $662 \mathrm{keV}$ ranges between $6 \%$ and $10 \%$ at FWHM for all the crystals and the average value of the detection threshold is $100 \mathrm{keV}$. A time coincidence window of $10 \mathrm{~ns}$ is used between the S- and the A-detectors to select $\gamma$-ray hits corresponding to the same event. The add-back energy spectrum of the $S$ and $A$ layer in time coincidence is displayed in Fig. 8 and shows an average resolution of $9 \%$ FWHM at $662 \mathrm{keV}$.

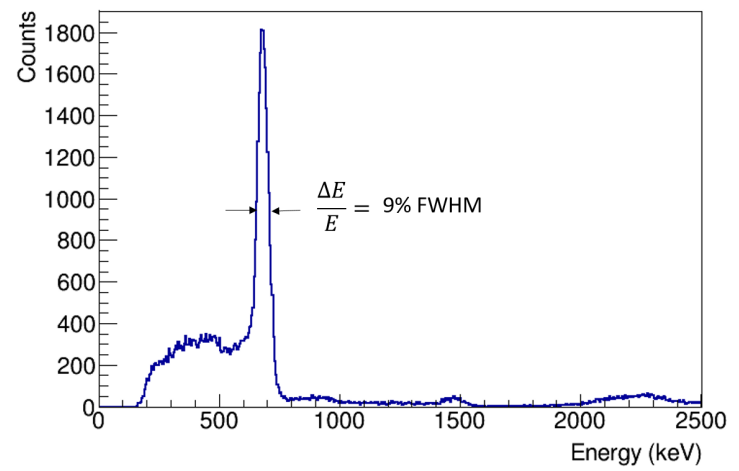

Figure 8: Sum spectrum for a ${ }^{137} \mathrm{Cs}$ source with the $S$ - and $A$-layers in time-coincidence. 
The intrinsic position reconstruction in each crystal is made by fitting the analytic expression of Li et al. [31] as described in Ref. [32]. Thus, an intrinsic position resolution of about $1 \mathrm{~mm}$ FWHM is achieved for both S- and A-detectors, respectively. The depth-of-interaction (DOI) in each crystal is obtained from the measured area at halfheight of the scintillation-photon distribution for each registered event. The present uncertainty on DOI is of about $5 \mathrm{~mm}$, as described in Ref. [32].

\subsection{Backprojection method}

A simple backprojection method has been implemented in order to reconstruct the Compton image at the plane of the $\gamma$-ray source. For illustrative purposes a point-like ${ }^{22} \mathrm{Na}$ source with an activity of $416 \mathrm{kBq}$ was centered at a distance of $165 \mathrm{~mm}$ in front of i-TED. The latter was operated with a $S-A$ separation length of $d_{f}=30 \mathrm{~mm}$ during a measuring time of $900 \mathrm{~s}$. The image was formed by defining a pixelated plane at $165 \mathrm{~mm}$ in front of the detector with squared voxels of $5 \mathrm{~mm}$ size. The latter were then filled with the geometric intersection of each Compton cone with the plane, following a similar approach as the one reported in Ref. [48]. The result obtained for the 2D-image is displayed in Fig. 9. A selection of \pm 4 pixels, corresponding to $\pm 20 \mathrm{~mm}$ was made around the maximum of the reconstructed distribution in order to analyze the image projection over the $x$ - and $y$-coordinates. The width of these projections is used below in Sec. 4.3 as an estimate for the image resolution.

\subsection{Efficiency versus $S$-A separation length $d_{f}$}

The efficiency for $662 \mathrm{keV} \gamma$-rays was measured as a function of the distance between the $S$ - and $A$-layers. To this aim a point-like ${ }^{137} \mathrm{Cs}$ source with an activity of $210.4 \mathrm{kBq}$ was placed at a distance of $165 \mathrm{~mm}$ from the center of the Compton camera. The efficiency was determined as a function of the distance between scatter and absorber detection planes by performing a series of measurements in steps of $\Delta d_{f}=2 \mathrm{~mm}$. Each measurement lasted for 15 minutes. The results are displayed in Fig. 10 and show a dependency with $d_{f}(\mathrm{~mm})$ given by $\varepsilon_{\gamma}=$ $1.358(14) \times 10^{-2}-2.52(7) \times 10^{-4} \cdot d_{f}+1.41(8) \times 10^{-6} \cdot d_{f}^{2}$ $(\%)$. For comparative purposes the efficiency extrapolated at a distance of $d_{s}=30 \mathrm{~mm}$ is also shown on the righthand side vertical axis. This will be used later in Sec. 5 for the discussion of the results.

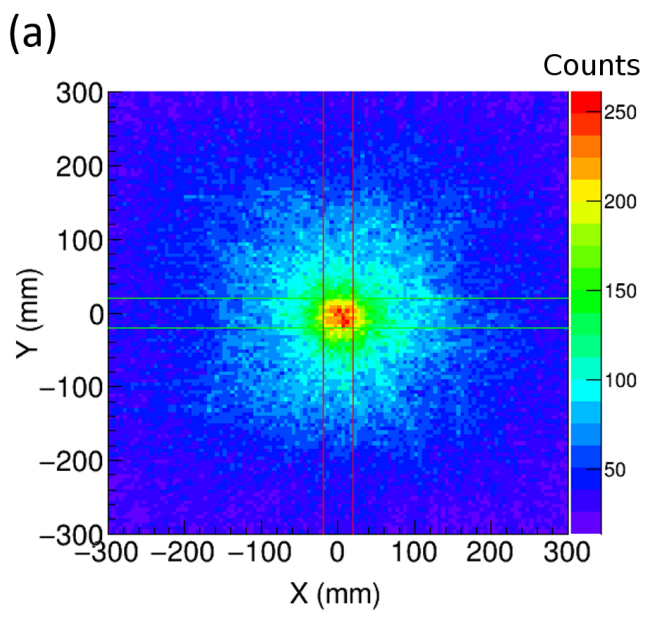

\section{(b)}

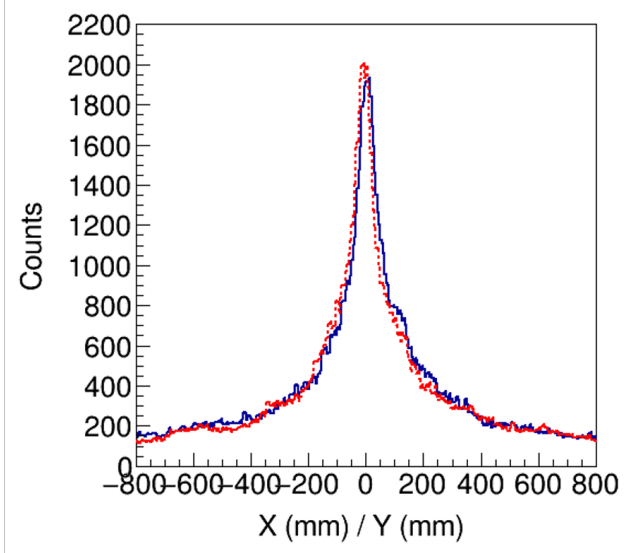

Figure 9: Backprojected 2D-image (a) for a $511 \mathrm{keV} \gamma$-rays from a ${ }^{22} \mathrm{Na}$ source centered at $165 \mathrm{~mm}$ in front of i-TED. Projections over the $\mathrm{x}$ - and $\mathrm{y}$-axis are shown in panel (b). 


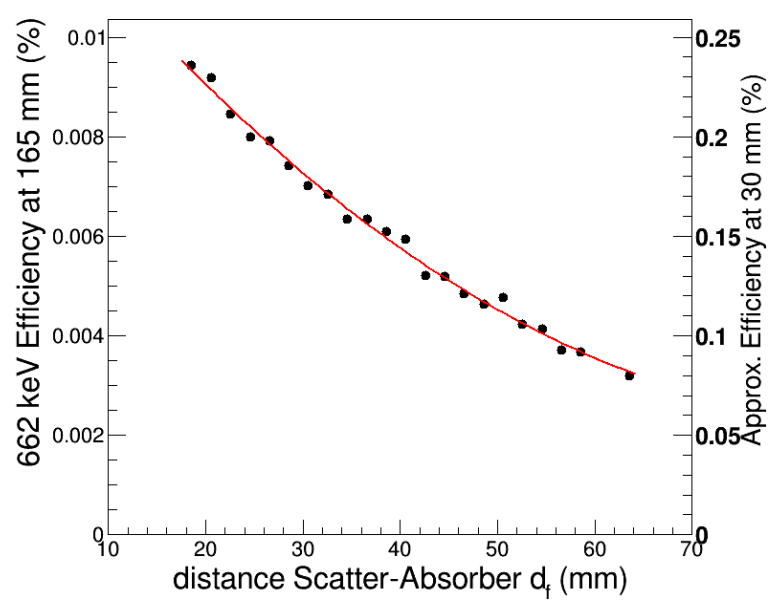

Figure 10: Measured efficiency for a point-like ${ }^{137} \mathrm{Cs}$ source $(662 \mathrm{keV})$ centered at $165 \mathrm{~mm}$ in front of i-TED. The right-axis shows the efficiency estimated for a distance of $30 \mathrm{~mm}$.

\subsection{Angular resolution versus $S-A$ separation length $d_{f}$}

The series of measurements described in the previous section were analyzed with the backprojection method and converted to angular units in order to determine the Angular Resolution Measure (ARM) to the level of one standard deviation as a function of the $S-A$ distance $d_{f}$. The results are displayed in Fig. 11. The angular reso-

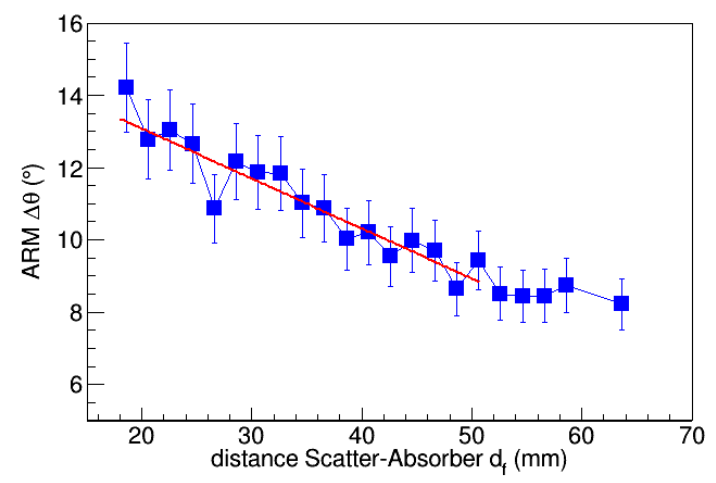

Figure 11: Measured angular resolution as a function of the $S-A$ separation length $d_{f}$ using the backprojection method for a point-like ${ }^{137} \mathrm{Cs}$ source $(662 \mathrm{keV})$ placed at $165 \mathrm{~mm}$ in front of the center of iTED. lution shows an approximate linear behavior as a function of the distance $d_{f}$, with ARM values varying from about $14^{\circ}$ to $9^{\circ}$ for $d_{f}$ lengths of $20 \mathrm{~mm}$ and $50 \mathrm{~mm}$, respectively. In this range, the ARM is described by $\Delta \theta=15.8(9)-0.14(2) \cdot d_{f}$ with $d_{f}$ in mm and ARM in degrees. Beyond $d_{f}=50 \mathrm{~mm}$ the angular resolution tends to a constant value of $8.5(3)^{\circ}$. This trend is expected from the rather constant resolution shown in the diagrams of Fig. 1 at large $d_{f}$ distances.

\subsection{Field of view}

In order to explore the field of view of i-TED a measurement was carried out placing the ${ }^{22} \mathrm{Na}$ source at nine different positions in front of the imager forming a cross with a spacing of $150 \mathrm{~mm}$. To prevent human error and eliminate any uncerainty related to the positioning of the source with respect to the imager a vertical gantry was setup as shown in Fig. 12 using LRT1500AL linear stages from Zaber Technologies Inc. The latter have an accuracy of $375 \mu \mathrm{m}$ and a repeteability error of $<2 \mu \mathrm{m}$.

The gantry was controlled and synchronized with our data-acquisition system, in a similar way as described in Ref. [32]. The distance between the central position and the imager was of $165 \mathrm{~mm}$. $S-A$ separation length of $d_{f}=30 \mathrm{~mm}$ was used in these measurements. The results for the reconstructed Compton images are shown in Fig. 13 both in Cartesian- and spherical coordinates. In the latter representation the zenith direction or reference for the $\theta$-coordinate coincides with the vertical $y$ direction of the cartesian coordinates, whereas the azimuthal angle $\phi$ is given with respect to the $x$-axis. In these figures a constant level background was determined and subtracted for each of the measured positions. Considering the simple backprojection reconstruction method implemented here the results are rather satisfactory because the reconstructed source positions coincide reasonably well with the true source positions in the main part of the field of view. A certain compression and loss of sensitivity can be appreciated in the peripheral positions. This effect can be most probably ascribed to the higher contribution of the peripheral region of the $S$ detector, where the linerarity is not as good as in the central region [32]. Better performances could be probably obtained by means of a more sophisticated method such as maximum-likelihood estimation [49, 50, 51]. However, this is out of the scope of the present work. Still, an angular field of view of about 
(a)

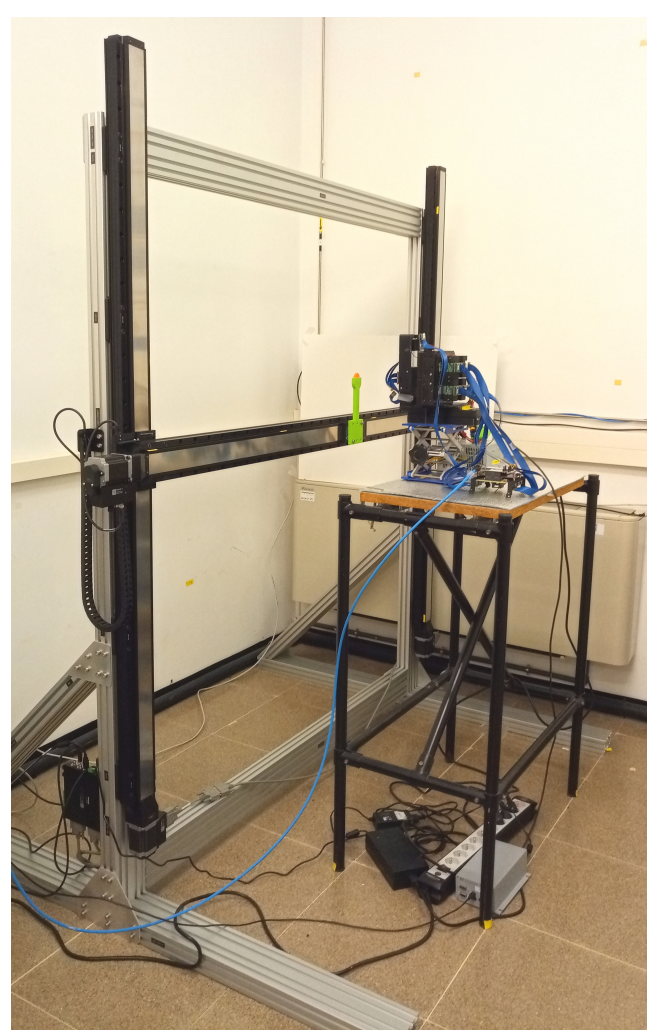

Figure 12: Set-up used to evaluate the field-of-view, with i-TED in front of the vertical positioning gantry.

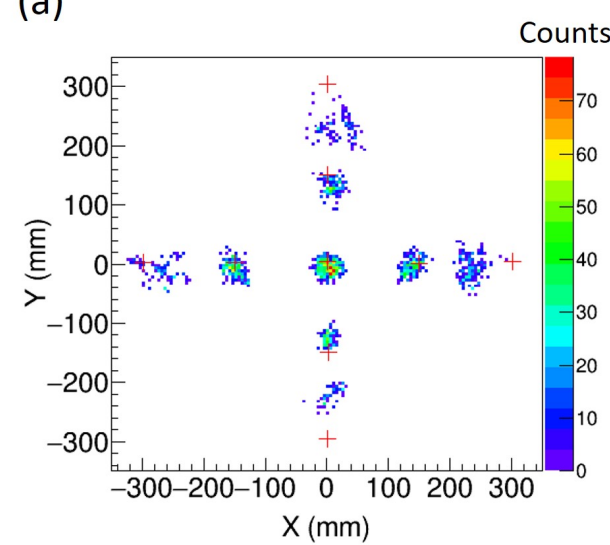

(b)

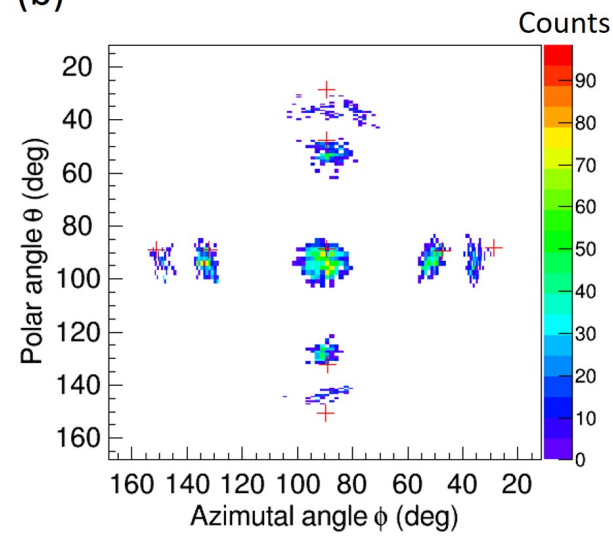

Figure 13: Positions reconstructed with the backprojection algorithm in Cartesian coordinates (a) and spherical coordinates (b). Small crossmarks indicate the true-positions of the source. 
$2 / 3$ of $2 \pi$ can be estimated from this measurement using the backprojection approach.

\section{Discussion and outlook}

i-TED is a high-efficiency Compton imager, which implements the new concept of Dynamic Electronic Collimation for enhanced fieldability and optimization of the measuring time. In this work an i-TED demonstrator based on five large $\mathrm{LaCl}_{3}(\mathrm{Ce})$ monolithic crystals and 320 readout channels has been assembled and characterized in terms of efficiency and imaging capabilities.

Efficiency is a key aspect for i-TED in order to keep the total measuring time within reasonable limits in neutron capture TOF experiments. Compared to other scintillatorbased Compton cameras with efficiencies reported in a similar energy range [44, 52], the efficiency found here is a factor of $\sim 50$ higher. This feature can be ascribed to the large sensitive volume of i-TED and the use of large and continuous (non-pixelated) scintillation crystals.

Angular resolutions between $8^{\circ}$ and $14^{\circ}$ have been obtained so far, which are quite satisfactory when considering the simple backprojection algorithm implemented. Such angular sensitivity should enable a significant reduction of the $\gamma$-ray background associated with external background sources in neutron capture TOF experiments [24]. The angular resolution found here is comparable to values reported recently, for example in Ref. [41, 52] for a similar $S-A$ separation of $d_{f} \sim 20 \mathrm{~mm}$. On the other hand, our resolution is approximately a factor of $\sim 2$ worse than the values reported in Ref. [44, 45] for similar $d_{f}$ values. This difference can be ascribed to the maximum likelihood expectation maximization (MLEM) method used in the latter work for the position reconstruction, and also to the better spectroscopic performance of their detectors.

There are several aspects related to the performance of i-TED which we plan to improve. One of them concerns the energy resolution of several of the detectors used, which was above the expected value due to a few channels missing in the readout electronics chain. An improved energy resolution will lead to a lower angular incertitude and improved overall imaging performance or, correspondingly, background rejection. Additionally, we plan to reduce the energy threshold of our $S$ detector, which in this work was at $100 \mathrm{keV}$. This should yield a better sensitivity to the Compton angles in the low range $\left(10^{\circ} \lesssim \theta \lesssim 30^{\circ}\right)$ of the high-resolution angular regime (see Fig 1). The i-TED background rejection algorithm conceived thus far for neutron capture TOF experiments is based on an analytic (backprojection) event-by-event decision, as described in Ref. [24]. In this respect we plan to explore the viability to combine more sophisticated maximum-likelihood expectation algorithms with the PHWT for the determination of the cross section. Finally, next steps include new measurements with i-TED using the pulsed neutron-beam of CNA-Seville [53], as a preliminary study before the commissioning at CERN $\mathrm{n}_{-}$TOF in 2021 after the long shutdown LS2. For the measurements at CNA we plan to implement ${ }^{6} \mathrm{LiH}$ neutron absorbers to reduce the intrinsic neutron sensitivity of i-TED, as discussed in Ref. [24]. By exposing the detector to the neutron beam we plan to perform a neutron sensitivity study similar to the one carried out in the past at FZK using $\mathrm{C}_{6} \mathrm{D}_{6}$ detectors [15].

\section{Declaration of competing interest}

The authors declare that they have no known competing financial interests or personal relationships that could have appeared to influence the work reported in this paper.

\section{CRediT authorship contribution statement}

V. Babiano: Software, Formal analysis, Investigation, Visualization. J. Balibrea: Visualization, Investigation, Writing -review \& editing. L. Caballero: Data curation, Investigation, Validation. D. Calvo: Resources, Investigation. I. Ladarescu: Software, Investigation, Formal analysis, Data curation. J. Lerendegui: Visualization, Investigation, Writing -review \& editing. S. Mira Prats: Methodology, Investigation. C. Domingo-Pardo: Conceptualization, Methodology, Supervision, Project administration, Writing - original draft, Writing -review \& editing, Funding acquisition, Software, Investigation.

\section{Acknowledgment}

This work has received funding from the European Research Council (ERC) under the European Union's Horizon 2020 research and innovation programme (ERC 
Consolidator Grant project HYMNS, with grant agreement $n^{\circ}$ 681740). The authors acknowledge support from the Spanish Ministerio de Ciencia e Innovación under grants FPA2014-52823-C2-1-P, FPA2017-83946-C2-1-P, CSIC for funding PIE-201750I26 and the program Severo Ochoa (SEV-2014-0398).

[1] E. Margaret Burbidge, G. R. Burbidge, William A. Fowler, and F. Hoyle. Synthesis of the elements in stars. Rev. Mod. Phys., 29:547-650, Oct 1957.

[2] A. G. W. Cameron. On the origin of the heavy elements. Astronomical Journal, 62:9-10, Feb 1957.

[3] F. Käppeler, R. Gallino, S. Bisterzo, and Wako Aoki. The s process: Nuclear physics, stellar models, and observations. Reviews of Modern Physics, 83(1):157-194, Jan 2011.

[4] N. Nishimura, R. Hirschi, T. Rauscher, A. St. J. Murphy, and G. Cescutti. Uncertainties in sprocess nucleosynthesis in massive stars determined by Monte Carlo variations. Monthly Notices of the Royal Astronomical Society, 469(2):1752-1767, Aug 2017.

[5] G. Cescutti, R. Hirschi, N. Nishimura, J. W. den Hartogh, T. Rauscher, A. St J. Murphy, and S. Cristallo. Uncertainties in s-process nucleosynthesis in low-mass stars determined from Monte Carlo variations. Monthly Notices of the Royal Astronomical Society, 478(3):4101-4127, Aug 2018.

[6] K. Wisshak, F. Kappeler, F. Voss, and K. Guber. The Karlsruhe 4 pi BaF/sub 2/ detector. IEEE Transactions on Nuclear Science, 36:101-105, February 1989.

[7] R. Reifarth, E.-I. Esch, A. Alpizar-Vicente, E. M. Bond, T. A. Bredeweg, S. E. Glover, U. Greife, R. Hatarik, R. C. Haight, A. Kronenberg, J. M. O’Donnell, R. S. Rundberg, J. M. Schwantes, J. L. Ullmann, D. J. Vieira, J. B. Wilhelmy, and J. M. Wouters. (n, $\gamma$ ) measurements on radioactive isotopes with DANCE. Nuclear Instruments and Methods in Physics Research B, 241:176-179, December 2005.
[8] C. Guerrero, D. Cano-Ott, E. Mendoza, J. L. Taín, A. Algora, E. Berthoumieux, N. Colonna, C. Domingo-Pardo, E. González-Romero, M. Heil, D. Jordán, F. Käppeler, C. Lampoudis, T. Martínez, C. Massimi, R. Plag, and n TOF Collaboration. Monte Carlo simulation of the $\mathrm{n}_{-}$TOF Total Absorption Calorimeter. Nuclear Instruments and Methods in Physics Research A, 671:108-117, 2012.

[9] R. L. Macklin and J. H. Gibbons. Capture-CrossSection Studies for 30-220-keV Neutrons Using a New Technique. Physical Review, 159:1007-1012, July 1967.

[10] U. Abbondanno, G. Aerts, H. Alvarez, S. Andriamonje, A. Angelopoulos, P. Assimakopoulos, C. O. Bacri, G. Badurek, P. Baumann, F. Bečvár, H. Beer, J. Benlliure, B. Berthier, E. Berthomieux, S. Boffi, C. Borcea, E. Boscolo-Marchi, N. Bustreo, P. Calviño, D. Cano-Ott, R. Capote, P. Carlson, P. Cennini, V. Chepel, E. Chiaveri, C. Coceva, N. Colonna, G. Cortes, D. Cortina, A. Couture, J. Cox, S. Dababneh, M. Dahlfors, S. David, R. Dolfini, C. Domingo-Pardo, I. Duran, C. Eleftheriadis, M. Embid-Segura, L. Ferrant, A. Ferrari, L. Ferreira-Lourenco, R. Ferreira-Marques, H. Frais-Koelbl, W. I. Furman, Y. Giomataris, I. F. Goncalves, E. Gonzalez-Romero, A. Goverdovski, F. Gramegna, E. Griesmayer, F. Gunsing, R. Haight, M. Heil, A. Herrera-Martinez, K. G. Ioannides, N. Janeva, E. Jericha, F. Käppeler, Y. Kadi, D. Karamanis, A. Kelic, V. Ketlerov, G. Kitis, P. E. Koehler, V. Konovalov, E. Kossionides, V. Lacoste, H. Leeb, A. Lindote, M. I. Lopes, M. Lozano, S. Lukic, S. Markov, S. Marrone, J. Martinez-Val, P. Mastinu, A. Mengoni, P. M. Milazzo, E. Minguez, A. MolinaCoballes, C. Moreau, F. Neves, H. Oberhummer, S. O'Brien, J. Pancin, T. Papaevangelou, C. Paradela, A. Pavlik, P. Pavlopoulos, A. Perez-Parra, J. M. Perlado, L. Perrot, V. Peskov, R. Plag, A. Plompen, A. Plukis, A. Poch, A. Policarpo, C. Pretel, J. M. Quesada, M. Radici, S. Raman, W. Rapp, T. Rauscher, R. Reifarth, F. Rejmund, M. Rosetti, C. Rubbia, G. Rudolf, P. Rullhusen, J. Salgado, E. Savvidis, J. C. Soares, C. Stephan, G. Tagliente, J. L. Tain, C. Tapia, L. Tassan-Got, 
L. M. N. Tavora, R. Terlizzi, M. Terrani, N. Tsangas, G. Vannini, P. Vaz, A. Ventura, D. VillamarinFernandez, M. Vincente-Vincente, V. Vlachoudis, R. Vlastou, F. Voss, H. Wendler, M. Wiescher, K. Wisshak, L. Zanini, and n TOF Collaboration. New experimental validation of the pulse height weighting technique for capture cross-section measurements. Nuclear Instruments and Methods in Physics Research A, 521:454-467, April 2004.

[11] A. Borella, G. Aerts, F. Gunsing, M. Moxon, P. Schillebeeckx, and R. Wynants. The use of $\mathrm{C}_{6} \mathrm{D}_{6}$ detectors for neutron induced capture cross-section measurements in the resonance region. Nuclear Instruments and Methods in Physics Research A, 577:626-640, July 2007.

[12] R. R. Winters and R. L. Macklin. Maxwellianaveraged Neutron Capture Cross Sections for 99Tc and 95-98Mo. The Astroph. Jour., 313:808, Feb 1987.

[13] U. Abbondanno, G. Aerts, F. Alvarez-Velarde, H. Álvarez-Pol, S. Andriamonje, J. Andrzejewski, G. Badurek, P. Baumann, F. Bečvář, J. Benlliure, E. Berthoumieux, F. Calviño, D. CanoOtt, R. Capote, P. Cennini, V. Chepel, E. Chiaveri, N. Colonna, G. Cortes, D. Cortina, A. Couture, J. Cox, S. Dababneh, M. Dahlfors, S. David, R. Dolfini, C. Domingo-Pardo, I. Duran, M. EmbidSegura, L. Ferrant, A. Ferrari, R. FerreiraMarques, H. Frais-Koelbl, W. Furman, I. Goncalves, R. Gallino, E. Gonzalez-Romero, A. Goverdovski, F. Gramegna, E. Griesmayer, F. Gunsing, B. Haas, R. Haight, M. Heil, A. Herrera-Martinez, S. Isaev, E. Jericha, F. Käppeler, Y. Kadi, D. Karadimos, M. Kerveno, V. Ketlerov, P. Koehler, V. Konovalov, M. Krtička, C. Lamboudis, H. Leeb, A. Lindote, I. Lopes, M. Lozano, S. Lukic, J. Marganiec, S. Marrone, J. Martinez-Val, P. Mastinu, A. Mengoni, P. M. Milazzo, A. Molina-Coballes, C. Moreau, M. Mosconi, F. Neves, H. Oberhummer, S. O'Brien, J. Pancin, T. Papaevangelou, C. Paradela, A. Pavlik, P. Pavlopoulos, J. M. Perlado, L. Perrot, M. Pignatari, R. Plag, A. Plompen, A. Plukis, A. Poch, A. Policarpo, C. Pretel, J. Quesada, S. Raman, W. Rapp, T. Rauscher, R. Reifarth, M. Rosetti,
C. Rubbia, G. Rudolf, P. Rullhusen, J. Salgado, J. C. Soares, C. Stephan, G. Tagliente, J. Tain, L. TassanGot, L. Tavora, R. Terlizzi, G. Vannini, P. Vaz, A. Ventura, D. Villamarin, M. C. Vincente, V. Vlachoudis, F. Voss, H. Wendler, M. Wiescher, and K. Wisshak. Neutron capture cross section measurement of ${ }^{151} \mathrm{Sm}$ at the cern neutron time of flight facility (n_tof). Phys. Rev. Lett., 93:161103, Oct 2004.

[14] C. Lederer, C. Massimi, S. Altstadt, J. Andrzejewski, L. Audouin, M. Barbagallo, V. Bécares, F. Bečváŕ, F. Belloni, E. Berthoumieux, J. Billowes, V. Boccone, D. Bosnar, M. Brugger, M. Calviani, F. Calviño, D. Cano-Ott, C. Carrapiço, F. Cerutti, E. Chiaveri, M. Chin, N. Colonna, G. Cortés, M. A. Cortés-Giraldo, M. Diakaki, C. DomingoPardo, I. Duran, R. Dressler, N. Dzysiuk, C. Eleftheriadis, A. Ferrari, K. Fraval, S. Ganesan, A. R. García, G. Giubrone, M. B. Gómez-Hornillos, I. F. Gonçalves, E. González-Romero, E. Griesmayer, C. Guerrero, F. Gunsing, P. Gurusamy, D. G. Jenkins, E. Jericha, Y. Kadi, F. Käppeler, D. Karadimos, N. Kivel, P. Koehler, M. Kokkoris, G. Korschinek, M. Krtička, J. Kroll, C. Langer, H. Leeb, L. S. Leong, R. Losito, A. Manousos, J. Marganiec, T. Martínez, P. F. Mastinu, M. Mastromarco, M. Meaze, E. Mendoza, A. Mengoni, P. M. Milazzo, F. Mingrone, M. Mirea, W. Mondelaers, C. Paradela, A. Pavlik, J. Perkowski, M. Pignatari, A. Plompen, J. Praena, J. M. Quesada, T. Rauscher, R. Reifarth, A. Riego, F. Roman, C. Rubbia, R. Sarmento, P. Schillebeeckx, S. Schmidt, D. Schumann, G. Tagliente, J. L. Tain, D. Tarrío, L. Tassan-Got, A. Tsinganis, S. Valenta, G. Vannini, V. Variale, P. Vaz, A. Ventura, R. Versaci, M. J. Vermeulen, V. Vlachoudis, R. Vlastou, A. Wallner, T. Ware, M. Weigand, C. Weiß, T. J. Wright, and P. Žugec. Neutron Capture Cross Section of Unstable Ni63: Implications for Stellar Nucleosynthesis. Phys. Rev. Lett., 110(2):022501, Jan 2013.

[15] R. Plag, M. Heil, F. Käppeler, P. Pavlopoulos, R. Reifarth, K. Wisshak, and n TOF Collaboration. An optimized $\mathrm{C}_{6} \mathrm{D}_{6}$ detector for studies of resonance-dominated ( $\mathrm{n}, \gamma)$ cross-sections. Nuclear Instruments and Methods in Physics Research A, 496:425-436, January 2003. 
[16] P. Žugec, N. Colonna, D. Bosnar, S. Altstadt, J. Andrzejewski, L. Audouin, M. Barbagallo, V. Bécares, F. Bečvár, F. Belloni, E. Berthoumieux, J. Billowes, V. Boccone, M. Brugger, M. Calviani, F. Calviño, D. Cano-Ott, C. Carrapiço, F. Cerutti, E. Chiaveri, M. Chin, G. Cortés, M. A. Cortés-Giraldo, M. Diakaki, C. Domingo-Pardo, R. Dressler, I. Duran, N. Dzysiuk, C. Eleftheriadis, A. Ferrari, K. Fraval, S. Ganesan, A. R. García, G. Giubrone, M. B. Gómez-Hornillos, I. F. Gonçalves, E. GonzálezRomero, E. Griesmayer, C. Guerrero, F. Gunsing, P. Gurusamy, S. Heinitz, D. G. Jenkins, E. Jericha, Y. Kadi, F. Käppeler, D. Karadimos, N. Kivel, P. Koehler, M. Kokkoris, M. Krtička, J. Kroll, C. Langer, C. Lederer, H. Leeb, L. S. Leong, S. Lo Meo, R. Losito, A. Manousos, J. Marganiec, T. Martínez, C. Massimi, P. F. Mastinu, M. Mastromarco, M. Meaze, E. Mendoza, A. Mengoni, P. M. Milazzo, F. Mingrone, M. Mirea, W. Mondalaers, C. Paradela, A. Pavlik, J. Perkowski, A. Plompen, J. Praena, J. M. Quesada, T. Rauscher, R. Reifarth, A. Riego, F. Roman, C. Rubbia, R. Sarmento, A. Saxena, P. Schillebeeckx, S. Schmidt, D. Schumann, G. Tagliente, J. L. Tain, D. Tarrío, L. TassanGot, A. Tsinganis, S. Valenta, G. Vannini, V. Variale, P. Vaz, A. Ventura, R. Versaci, M. J. Vermeulen, V. Vlachoudis, R. Vlastou, A. Wallner, T. Ware, M. Weigand, C. Weiß, and T. Wright. GEANT4 simulation of the neutron background of the $\mathrm{C}_{6} \mathrm{D}_{6}$ set-up for capture studies at n_TOF. Nuclear Instruments and Methods in Physics Research A, 760:5767, October 2014.

[17] G. Tagliente, P. M. Milazzo, K. Fujii, U. Abbondanno, G. Aerts, H. Álvarez, F. Alvarez-Velarde, S. Andriamonje, J. Andrzejewski, L. Audouin, G. Badurek, P. Baumann, F. Bečváŕ, F. Belloni, E. Berthoumieux, F. Calviño, M. Calviani, D. Cano-Ott, R. Capote, C. Carrapiço, P. Cennini, V. Chepel, E. Chiaveri, N. Colonna, G. Cortes, A. Couture, M. Dahlfors, S. David, I. Dillmann, C. Domingo-Pardo, W. Dridi, I. Duran, C. Eleftheriadis, M. Embid-Segura, A. Ferrari, R. FerreiraMarques, W. Furman, I. Goncalves, E. GonzalezRomero, F. Gramegna, C. Guerrero, F. Gunsing, B. Haas, R. Haight, M. Heil, A. Herrera-Martinez,
E. Jericha, F. Käppeler, Y. Kadi, D. Karadimos, D. Karamanis, M. Kerveno, E. Kossionides, M. Krtička, C. Lamboudis, H. Leeb, A. Lindote, I. Lopes, S. Lukic, J. Marganiec, S. Marrone, T. Martínez, C. Massimi, P. Mastinu, A. Mengoni, C. Moreau, M. Mosconi, F. Neves, H. Oberhummer, S. O'Brien, C. Papachristodoulou, C. Papadopoulos, C. Paradela, N. Patronis, A. Pavlik, P. Pavlopoulos, L. Perrot, M. T. Pigni, R. Plag, A. Plompen, A. Plukis, A. Poch, J. Praena, C. Pretel, J. Quesada, R. Reifarth, M. Rosetti, C. Rubbia, G. Rudolf, P. Rullhusen, J. Salgado, C. Santos, L. Sarchiapone, I. Savvidis, C. Stephan, J. L. Tain, L. Tassan-Got, L. Tavora, R. Terlizzi, G. Vannini, P. Vaz, A. Ventura, D. Villamarin, M. C. Vincente, V. Vlachoudis, R. Vlastou, F. Voss, S. Walter, M. Wiescher, and K. Wisshak. The ${ }^{93} \mathrm{Zr}(\mathrm{n}, \gamma)$ reaction up to $8 \mathrm{keV}$ neutron energy. Phys. Rev. C, 87(1):014622, Jan 2013.

[18] P. Neyskens, S. van Eck, A. Jorissen, S. Goriely, L. Siess, and B. Plez. The temperature and chronology of heavy-element synthesis in low-mass stars. Nature, 517(7533):174-176, Jan 2015.

[19] G. Walter, H. Beer, F. Kaeppeler, G. Reffo, and F. Fabbri. The s-process branching at Se-79. Astronomy and Astrophysics, 167(1):186-199, Oct 1986.

[20] D. L. Pérez Magán, L. Caballero, C. DomingoPardo, J. Agramunt-Ros, F. Albiol, A. Casanovas, A. González, C. Guerrero, J. Lerendegui-Marco, and A. Tarifeño-Saldivia. First tests of the applicability of $\gamma$-ray imaging for background discrimination in time-of-flight neutron capture measurements. $\mathrm{Nu}$ clear Instruments and Methods in Physics Research A, 823:107-119, July 2016.

[21] V. Schönfelder, A. Hirner, and K. Schneider. A telescope for soft gamma ray astronomy. Nuclear Instruments and Methods, 107:385-394, 1973.

[22] D.B. Everett, J.S.Fleming, R.W. Todd and J.M. Nightingale. Gamma-radiation imaging system based on the compton effect. Proc. IEEE, Vol. 124, pp.995-1000, 1977.

[23] High-sensitivitY Measurements of key stellar Nucleo-Synthesis reactions (HYMNS), ERC- 
Consolidator Grant Agreement No. 681740, PI: C. Domingo Pardo.

[24] C. Domingo-Pardo. i-TED: A novel concept for high-sensitivity (n, $\gamma$ ) cross-section measurements. Nuclear Instruments and Methods in Physics Research A, 825:78-86, July 2016.

[25] Volker Schnfelder. Lessons learnt from comptel for future telescopes. New Astronomy Reviews, 48(1): 193 - 198, 2004. Astronomy with Radioactivities IV and Filling the Sensitivity Gap in $\mathrm{MeV}$ Astronomy.

[26] Yuto Ichinohe, Yuusuke Uchida, Shin Watanabe, Ikumi Edahiro, Katsuhiro Hayashi, Takafumi Kawano, Masanori Ohno, Masayuki Ohta, Shin ichiro Takeda, Yasushi Fukazawa, Miho Katsuragawa, Kazuhiro Nakazawa, Hirokazu Odaka, Hiroyasu Tajima, Hiromitsu Takahashi, Tadayuki Takahashi, and Takayuki Yuasa. The first demonstration of the concept of "narrow-FOV Si/CdTe semiconductor Compton camera". Nuclear Instruments and Methods in Physics Research A, 806:513, Jan 2016.

[27] Hiroyasu Tajima, Shin Watanabe, Yasushi Fukazawa, Roger Bland ford, Teruaki Enoto, Andrea Goldwurm, Kouichi Hagino, Katsuhiro Hayashi, Yuto Ichinohe, Jun Kataoka, Jun'ichiro Katsuta, Takao Kitaguchi, Motohide Kokubun, Philippe Laurent, François Lebrun, Olivier Limousin, Grzegorz M. Madejski, Kazuo Makishima, Tsunefumi Mizuno, Kunishiro Mori, Takeshi Nakamori, Toshio Nakano, Kazuhiro Nakazawa, Hirofumi Noda, Hirokazu Odaka, Masanori Ohno, Masayuki Ohta, Shinya Saito, Goro Sato, Rie Sato, Shin'ichiro Takeda, Hiromitsu Takahashi, Tadayuki Takahashi, Takaaki Tanaka, Yasuyuki Tanaka, Yukikatsu Terada, Hideki Uchiyama, Yasunobu Uchiyama, Kazutaka Yamaoka, Yoichi Yatsu, Daisuke Yonetoku, and Takayuki Yuasa. Design and performance of Soft Gamma-ray Detector onboard the Hitomi (ASTROH) satellite. Journal of Astronomical Telescopes, Instruments, and Systems, 4:021411, Apr 2018.
[28] S. Aldawood, P. G. Thirolf, A. Miani, M. Böhmer, G. Dedes, R. Gernhäuser, C. Lang, S. Liprandi, L. Maier, T. Marinšek, M. Mayerhofer, D. R. Schaart, I. V. Lozano, and K. Parodi. Development of a Compton camera for prompt-gamma medical imaging. Radiation Physics and Chemistry, 140:190-197, November 2017.

[29] S. Tashenov and J. Gerl. Imaging algorithm for background suppression in the planned gamma tracking array of DESPEC. Nuclear Instruments and Methods in Physics Research A, 586(2):224 228, Feb 2008.

[30] L. Mihailescu, K.M. Vetter, M.T. Burks, E.L. Hull, and W.W. Craig. Speir: A ge compton camera. Nuclear Instruments and Methods in Physics Research Section A: Accelerators, Spectrometers, Detectors and Associated Equipment, 570(1):89 - 100, 2007.

[31] Z. Li, M. Wedrowski, P. Bruyndonckx, and G. Vandersteen. Nonlinear least-squares modeling of 3D interaction position in a monolithic scintillator block. Physics in Medicine and Biology, 55:65156532, November 2010.

[32] V. Babiano, L. Caballero, D. Calvo, I. Ladarescu, P. Olleros, and C. Domingo-Pardo. $\gamma$-Ray position reconstruction in large monolithic $\mathrm{LaCl}_{3}(\mathrm{Ce})$ crystals with SiPM readout. Nuclear Instruments and Methods in Physics Research A, 931:1-22, Jul 2019.

[33] A. Gostojić, V. Tatischeff, J. Kiener, C. Hamadache, J. Peyré, N. Karkour, D. Linget, L. Gibelin, X. Lafay, X. Grave, N. Dosme, E. Legay, S. Blin, and $\mathrm{P}$. Barrillon. Characterization of $\mathrm{LaBr}_{3}: \mathrm{Ce}$ and $\mathrm{CeBr}_{3}$ calorimeter modules for $3 \mathrm{D}$ imaging in gamma-ray astronomy. Nuclear Instruments and Methods in Physics Research A, 832:24-42, October 2016.

[34] S. Liprandi, M. Mayerhofer, S. Aldawood, T. Binder, G. Dedes, A. Miani, D. Schaart, I. Valencia Lozano, K. Parodi, and P. Thirolf. Sub-3mm spatial resolution from a large monolithic $\mathrm{LaBr} 3$ (Ce) scintillator. Current Directions in Biomedical Engineering, 3:655-659, February 2017. 
[35] A. Ulyanov, O. Morris, O. J. Roberts, I. Tobin, L. Hanlon, S. McBreen, D. Murphy, N. Nelms, and B. Shortt. Localisation of gamma-ray interaction points in thick monolithic $\mathrm{CeBr}_{3}$ and $\mathrm{LaBr}_{3}: \mathrm{Ce}$ scintillators. Nuclear Instruments and Methods in Physics Research A, 844:81-89, February 2017.

[36] P. Olleros, L. Caballero, C. Domingo-Pardo, V. Babiano, I. Ladarescu, D. Calvo, P. Gramage, E. Nacher, J. L. Tain, and A. Tolosa. On the performance of large monolithic $\mathrm{LaCl}_{3}(\mathrm{Ce})$ crystals coupled to pixelated silicon photosensors. Journal of Instrumentation, 13:P03014, March 2018.

[37] Roland Ribberfors. Relationship of the relativistic compton cross section to the momentum distribution of bound electron states. Phys. Rev. B, 12:20672074, Sep 1975.

[38] O. Klein and Y. Nishina. Über die streuung von strahlung durch freie elektronen nach der neuen relativistischen quantendynamik von dirac. Zeitschrift für Physik, 52(11):853-868, Nov 1929.

[39] C. Domingo Pardo, L. Caballero Ontanaya, F.J. Albiol Colomer, and J. Agramunt Ros. Focusable Compton Camera equipped with variable separation detectors. Patentscope, Dec 2015. PCT/ES2016/070916.

[40] H. C. Boston, J. Gillam, A. J. Boston, R. J. Cooper, J. Cresswell, A. N. Grint, A. R. Mather, P. J. Nolan, D. P. Scraggs, G. Turk, C. J. Hall, I. Lazarus, A. Berry, T. Beveridge, and R. Lewis. Orthogonal strip HPGe planar SmartPET detectors in Compton configuration. Nuclear Instruments and Methods in Physics Research A, 580:929-933, October 2007.

[41] J. Kataoka, A. Kishimoto, T. Nishiyama, T. Fujita, K. Takeuchi, T. Kato, T. Nakamori, S. Ohsuka, S. Nakamura, M. Hirayanagi, S. Adachi, T. Uchiyama, and K. Yamamoto. Handy Compton camera using 3D position-sensitive scintillators coupled with large-area monolithic MPPC arrays. Nuclear Instruments and Methods in Physics Research A, 732:403-407, December 2013.

[42] K. Takeuchi, J. Kataoka, T. Nishiyama, T. Fujita, A. Kishimoto, S. Ohsuka, S. Nakamura, S. Adachi,
M. Hirayanagi, T. Uchiyama, Y. Ishikawa, and T. Kato. "Stereo Compton cameras" for the 3-D localization of radioisotopes. Nuclear Instruments and Methods in Physics Research A, 765:187-191, Nov 2014.

[43] Taewoong Lee and Wonho Lee. Multiple scattering Compton camera with neutron activation for material inspection. Nuclear Instruments and Methods in Physics Research A, 784:423-429, Jun 2015.

[44] A. Kishimoto, J. Kataoka, A. Koide, K. Sueoka, Y. Iwamoto, T. Taya, and S. Ohsuka. Development of a compact scintillator-based high-resolution Compton camera for molecular imaging. Nuclear Instruments and Methods in Physics Research A, 845:656-659, Feb 2017.

[45] Enrique Muñoz, John Barrio, Ane Etxebeste, Pablo G Ortega, Carlos Lacasta, Josep F Oliver, Carles Solaz, and Gabriela Llosá. Performance evaluation of MACACO: a multilayer compton camera. Physics in Medicine $\mathcal{E}$ Biology, 62(18):7321-7341, aug 2017.

[46] Aya Kishimoto, Jun Kataoka, Takanori Taya, Leo Tagawa, Saku Mochizuki, Shinji Ohsuka, Yuto Nagao, Keisuke Kurita, Mitsutaka Yamaguchi, Naoki Kawachi, Keiko Matsunaga, Hayato Ikeda, Eku Shimosegawa, and Jun Hatazawa. First demonstration of multi-color 3-D in vivo imaging using ultra-compact Compton camera. Scientific Reports, 7:2110, May 2017.

[47] A. Di Francesco, R. Bugalho, L. Oliveira, A. Rivetti, M. Rolo, J. C. Silva, and J. Varela. TOFPET 2: A high-performance circuit for PET time-of-flight. Nuclear Instruments and Methods in Physics Research A, 824:194-195, July 2016.

[48] S. J. Wilderman, W. L. Rogers, G. F. Knoll, and J. C. Engdahl. Fast algorithm for list mode backprojection of Compton scatter camera data. IEEE Transactions on Nuclear Science, 45:957-962, June 1998.

[49] A. Sarmouk, A. D. Zych, T. O’Neill, R. S. White, and O. T. Tumer. Imaging gamma-ray sources with 
a Compton double scatter telescope using the expectation maximization maximum likelihood (EMML) technique. IEEE Transactions on Nuclear Science, 41(4):1349-1353, Aug 1994.

[50] L. Parra and H. H. Barrett. List-mode likelihood: Em algorithm and image quality estimation demonstrated on 2-d pet. IEEE Transactions on Medical Imaging, 17(2):228-235, April 1998.

[51] D. Xu and Z. He. Gamma-ray energy-imaging integrated spectral deconvolution. Nuclear Instruments and Methods in Physics Research A, 574:98-109, April 2007.

[52] Yuto Nagao, Mitsutaka Yamaguchi, Naoki Kawachi, and Hiroshi Watabe. Development of a costeffective Compton camera using a positron emission tomography data acquisition system. Nuclear Instruments and Methods in Physics Research A, 912:20-23, Dec 2018.

[53] M. Macas, B. Fernndez, and J. Praena. The first neutron time-of-flight line in spain: Commissioning and new data for the definition of a neutron standard field. Radiation Physics and Chemistry, 168:108538, 2020. 